\title{
The effect of shockwave profile shape on dynamic brittle failure
}

\author{
E.N. Brown ${ }^{1}$, J.P. Escobedo' ${ }^{2}$ C.P. Trujillo², and G.T. Gray III ${ }^{2}$ \\ 1 P-23, MS G-755, Los Alamos National Laboratory, Los Alamos, NM 87545, USA \\ 2 MST-8, MS G-755, Los Alamos National Laboratory, Los Alamos, NM 87545, USA
}

\begin{abstract}
The role of shock wave loading profile is investigated for the failure processes in a brittle material. The dynamic damage response of ductile metals has been demonstrated to be critically dependent on the shockwave profile and the stressstate of the shock. Changing from a square to triangular (Taylor) profile with an identical peak compressive stress has been reported to increase the "spall strength" by over a factor of two and suppress damage mechanisms. The spall strength of tungsten heavy alloy (WHA) based on plate impact square-wave loading has been extensively reported in the literature. Here a triangular wave loading profile is achieved with a composite flyer plate of graded density in contrast to the square-wave loading. Counter to the strong dependence in wave profile in ductile metals, for WHA, both square and triangle wave profiles the failure is by brittle cleavage fracture with additional energy dissipation through crack branching in the more brittle tungsten particles, largely indistinguishable between wave profiles. The time for crack nucleation is negligible compared to the duration of the experiment and the crack propagation rate is limited to the sound speed as defined by the shock velocity.
\end{abstract}

\section{Introduction}

In the current work we study the effect of shock wave loading profile on the failure processes in brittle tungsten heavy alloy. The last several decades have seen the dynamic tensile strength or spall strength of a wide range of materials characterized by flyer plate impact experiments. In its simplest form, the flyer plate experiment consists of a single monolithic impactor launched against a sample target to generate a square-wave loading profile for durations on the order of a microsecond. The real-time response of the sample target is probed by measuring the free surface velocity using a velocimetry interferometer for any surface reflector (VISAR) or a photon dopler velocimetry (PDV) systems. The resultant spall strength determined from the observed pull back signal has often been considered an absolute material property. However, for ductile metals it has been shown that the spall response is dependant on the pulse shape in addition to the peak stress. By changing the pulse shape from a square-wave loading profile to a high-explosive-loaded Taylor wave or composite flyer plate triangular wave of identical peak stress, the observed spall strength can increase significantly or tensile damage can be prohibited entirely.

Gray et al. [1] showed for 316 L stainless steel that shock hardening was relatively unchanged between triangular and square wave profiles at peak shock stresses of $6.6 \mathrm{GPa}$. Conversely, while the square-wave resulted in incipient spallation in $316 \mathrm{~L} \mathrm{SS}$ at a peak stress of $6.6 \mathrm{GPa}$, the triangular-wave loaded material exhibited no waveprofile "pull-back" nor damage evolution until a peak stress of at least $14.5 \mathrm{GPa}$ [2]. In $\mathrm{Cu}$ the shock response was more sensitive to loading profile [3]. While a square wave in $\mathrm{Cu}$ at $8 \mathrm{GPa}$ resulted in localized damage with sufficient ductile void coalescence to result in complete separation of a single spall scab, triangle-wave loading at the same peak stress revealed a consistent ringing in the particle velocity indicating formation of a free surface or a significant damage layer in the material but no voids were formed. The spall strength for the square and trianglewave loading were reported to be 1.28 and $2.04 \mathrm{GPa}$, respectively [4]. Mechanistically, the dependence on loading profile is coupled to the kinetics of ductile plastic deformation leading to void nucleation, growth, and subsequent linkage to form a spall plane. Within the tensile loaded region of the sample, the damage mechanisms require sufficient energy_from area under the loading profile - to be applied over a sufficiently long times to accommodate the kinetics of the deformation and damage evolution mechanisms. The effect of shock wave loading profile on brittle failure mechanisms has not previously been reported.

Tungsten heavy alloy (WHA) is a composite material of tungsten particles (body-centered-cubic) of approximately $50 \mu \mathrm{m}$ diameter in an austenitic (face-centeredcubic) matrix comprised of tungsten, nickel, and iron that exhibits brittle crack growth failure. The compite displays improved ductility under compression and tension in comparison to that of pure polycrystalline tungsten. The shock response based on plate impact square-wave loading has been extensively reported in the literature for pure polycrystalline tungsten [5-8] and tungsten heavy alloys [9-14].

Zurek and Gray [7] reported a spall strength of 3.4 GPa. Dandekar and Weisgerber [11] reported the spall strength and Hugoniot elastic limit (HEL) of WHA to be 1.72.0 $\mathrm{GPa}$ and $2.8 \mathrm{GPa}$, respectively for plate impact. Vogler and Clayton [14] employed line-VISAR to spatially resolve statistics on the spall strength of WHA based on plate impact, fitting their measurements to both normal and Weibull distributions. Across five WHA samples with different material processing and sample orientation, the average spall strength within a given sample varied from 1.66 to $2.13 \mathrm{GPa}$ with standard deviations from 0.31 to $0.61 \mathrm{GPa}$. They reported the HEL to vary from 0.83 to $4.09 \mathrm{GPa}$. Bless reported the spall strength for WHA to be $2.6 \mathrm{GPa}$ but did not observe an HEL. Vogler and Clayton [14] reported that higher velocity impacts produce 
a higher spall strength, suggesting that the regions where failure occurs (e.g., W-matrix interfaces and $\mathrm{W}-\mathrm{W}$ grain boundaries) are preconditioned to a more durable state by the initial compressive pulse. Dandekar also showed some variation of spall strength with impact velocity for WHA [11], as well as in other brittle materials [15]. Chang and Choi [16] employed RDX to explosively load WHA, imparting a Taylor wave loading profile, and reported significantly higher spall strength and HEL values of 3.74 to $5.60 \mathrm{GPa}$ and $3.19 \mathrm{GPa}$ respectively. However, Baoping et al. [17] also employed explosive loading and reported spall strength and HEL values of 0.54 to $3.04 \mathrm{GPa}$ and 2.0 to $3.3 \mathrm{GPa}$ respectively, which is not statistically higher than reported in literature for plate impact.

The current work investigates spall strength as a function of triangular wave loading profile achieved with a composite flyer plate of graded density in contrast to the square-wave loading profile imparted via the impact of a classic monolithic flyer plate. The targets incorporate momentum trapping to prevent edge release waves from imparting complicated loading on the samples and are soft captured. Detailed fractographic analysis of the damage evolution in spalled WHA samples as a function of shock wave profile of comparable peak stress is presented. For both square and triangle wave profiles, the failure is by brittle transparticle crack growth with additional energy dissipation through crack branching in the more brittle tungsten particles, and is largely indistinguishable between wave profiles. In this brittle material all relevant damage kinetics are equally supported by the square and triangular wave and the spall strength is shown to be dominated by the peak stress independent of loading profile.

\section{Experimental technique}

Soft recovered spall plate impact experiments were carried out using the $80 \mathrm{~mm}$ bore gas launcher previously presented by Gray [18]. Two identical $4 \mathrm{~mm}$ thick right circular WHA targets were prepared with press fit momentum trapping rings to mitigate perturbations from edge release waves. A square wave profile was achieved using a $2 \mathrm{~mm}$ thick WHA monolithic impactor. The relative thickness of the target and impactor was chosen to locate the spall plane at the midline of the target. A triangular wave profile was achieved with a composite projectile of $0.5 \mathrm{~mm}$ of $\mathrm{W}$. Impact velocity was measured to an accuracy of $1 \%$ using a sequential pressure transducer method and sample tilt was fixed to $\sim 1$ mrad by means of an adjustable specimen mount. Both experiments were executed to achieve a peak stress of $\sim 15.5 \mathrm{GPa}$, which is significantly above the Hugoniot elastic limit (HEL) of WHA.

The free surface velocity (FSV) profiles were measured using PDV (photon doppler velocimetry) singlepoint probes. The resultant spall strengths for the two experiments are determined as proposed by Novikov [19] and the correction proposed by Kanel by [20,21],

$$
\sigma_{\text {spall }}=\frac{1}{2} \rho_{0} c_{B}(\Delta F S V+\delta)
$$

where $\rho_{0}$ is the ambient density $\left(19.256 \mathrm{~g} / \mathrm{cm}^{3}\right), C_{B}$ is the bulk sound speed $(4.014 \mathrm{~mm} / \mu \mathrm{s})$, and $\triangle F S V$ is the

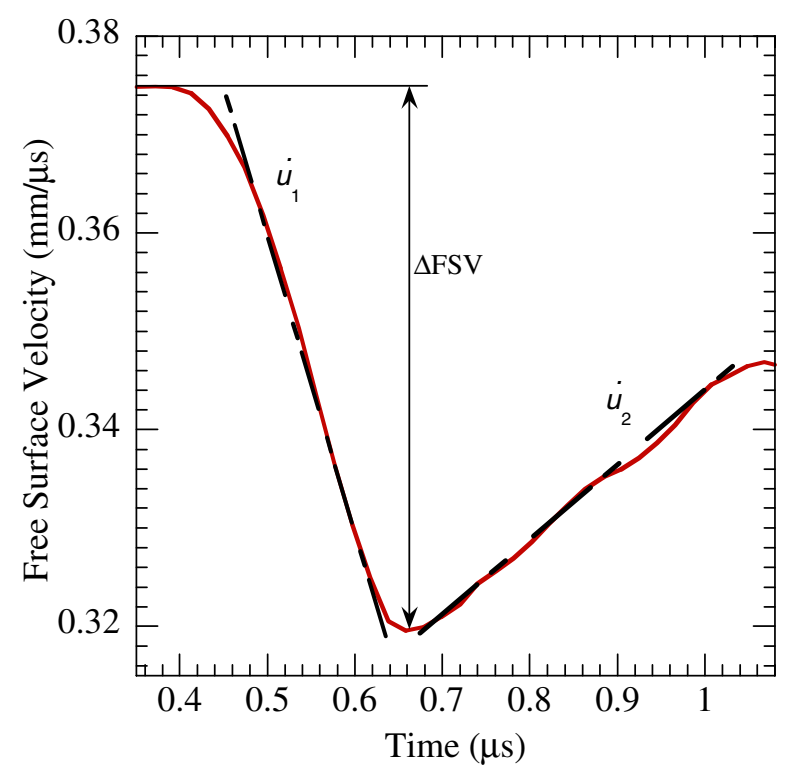

Fig. 1. The decompression rate and the re-compression rates on the spall pull-back signal illustrated with the square wave profile.

observed pull back signal (as shown in figure 1). The accuracy of the spall strength is improved by correcting for the FSV

$$
\delta=h\left(\frac{1}{C_{B}}-\frac{1}{C_{L}}\right) * \frac{\left|\dot{u}_{1} * \dot{u}_{2}\right|}{\left|\dot{u}_{1}\right|+\dot{u}_{2}},
$$

where $h$ is the thickness of the spalled region, $C_{L}$ is the longitudinal sound speed $(5.22 \mathrm{~mm} / \mu \mathrm{s})$, and the decompression rate and the re-compression rates are

$$
\left|\dot{u}_{1}\right|=-1 / 2 \frac{\mathrm{dFSV}}{\mathrm{dt}} \text { and } \dot{u}_{2}=1 / 2 \frac{\mathrm{dFSV}}{\mathrm{dt}}
$$

respectively.

\section{Results and discussion}

The free surface velocity profiles measured with PDV are show in figure 2. Both the square and triangularwave profiles exhibit a slight inflection in the shock front at $\sim 0.1 \mathrm{~mm} / \mu \mathrm{s}$, potentially indicative of the HEL. Although the square-wave profile exhibits some rounding and increases slightly with time, the stress pulse remains within $95 \%$ of the peak stress for $0.52 \mu \mathrm{s}$, before starting to release, exhibited a classis spall pull-back signal and ringing. In contrast, the triangular wave profile exhibits a sharp transition at peak stress and remains within $95 \%$ of the peak stress for less than $0.08 \mu \mathrm{s}$, before starting to release and exhibited a classis spall pull-back signal and ringing. The pull-back region of the free surface velocity profiles are show in figure 3 . The free surface velocity pull-backs are 0.056 and $0.067 \mathrm{~mm} / \mu \mathrm{s}$ for the square and triangular wave profiles respectively. Using Eq. (1) these measurements result in spall strengths of 2.16 and $2.59 \mathrm{GPa}$ for the square and triangular wave profiles respectively. While there is a $\sim 16 \%$ difference in spall strengths, there is notable difference in the shape 


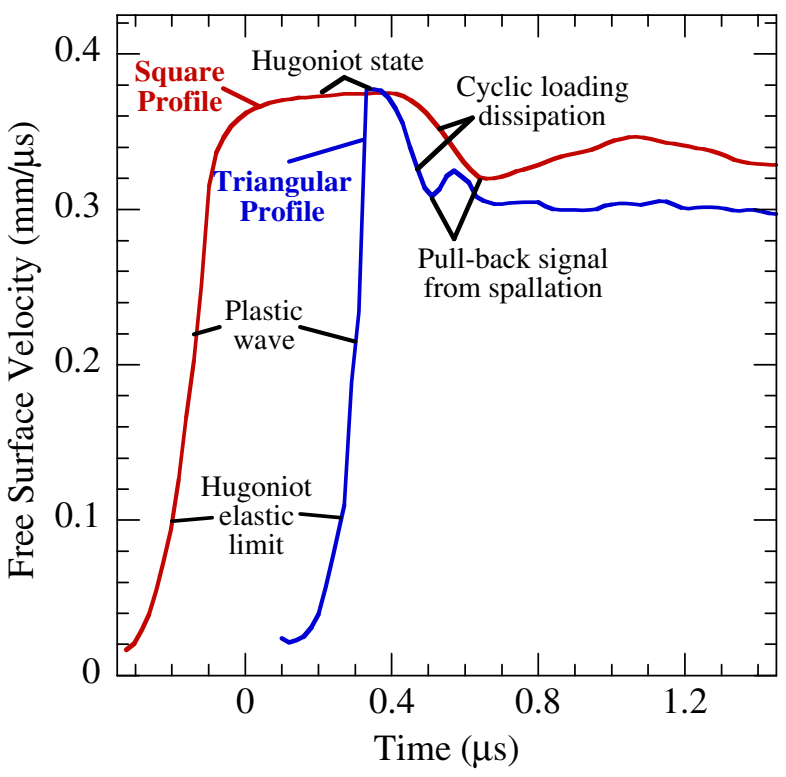

Fig. 2. PDV traces of the free surface velocity showing shock loading with square and triangular wave forms and pull back signal indicative of spall plane formation. The curves are plotted such that the drop from the peak state starts at similar times for both shots.

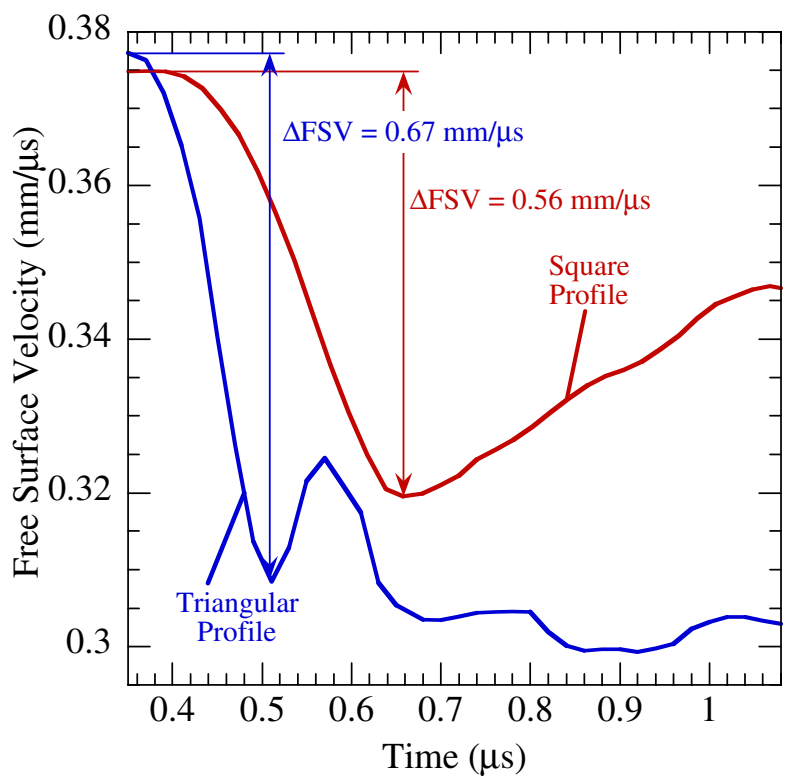

Fig. 3. PDV traces of the free surface velocity highlighting the pull back signal indicative of spall plane formation.

of the pullback wave. Both the decompression rate and the re-compression rates after the minima are different, as illustrated in figure 3.

Further correcting the spall strength calculations with Eq. (2) for the thickness of the spalled region from figure 4, the spall strength values are 2.23 and $2.64 \mathrm{GPa}$ for the square and triangular wave profiles respectively, reducing the difference to $\sim 15 \%$. These spall strength values are consistent with the majority of values reported in the literature review in the introduction. It is worth noting that the difference between the two specimens is less than the sample-to-sample scatter generally reported for WHA and

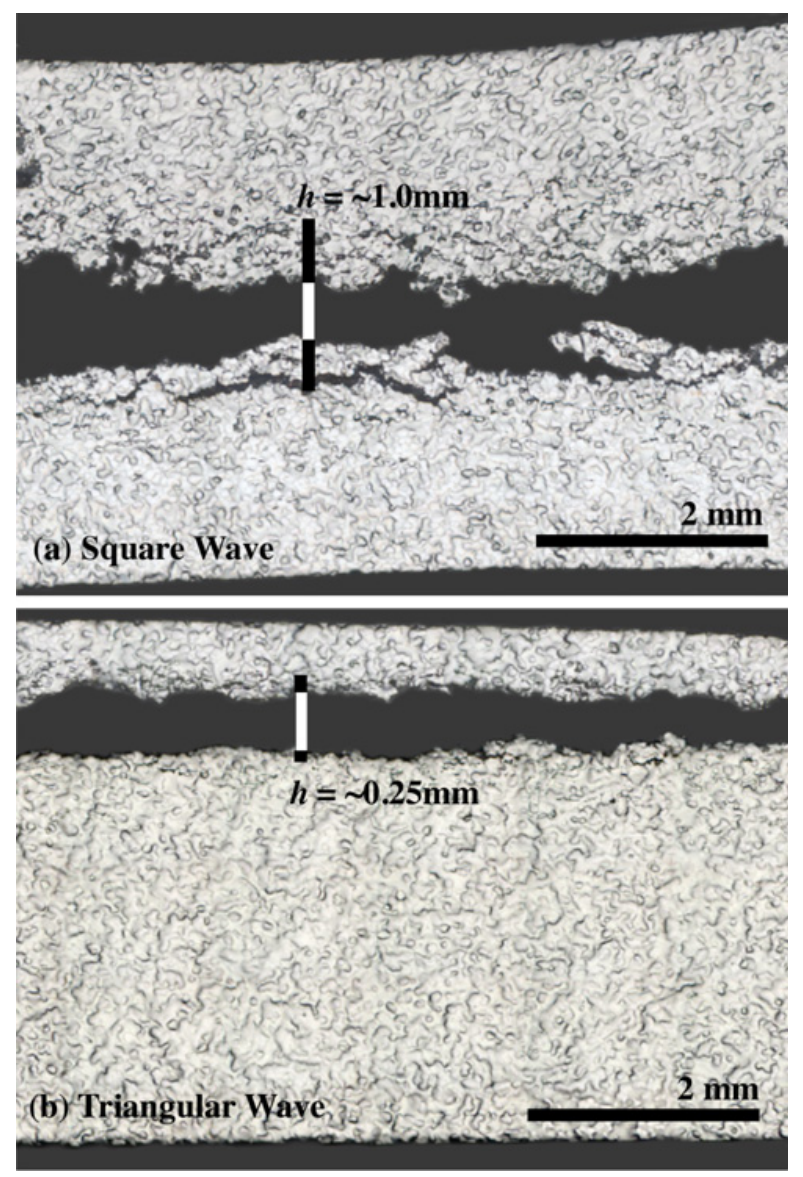

Fig. 4. Optical micrographs of the (a) square-wave and (b) trangle-wave loaded spalled samples. The shock direction is from bottom to top. The spall plane is significantly rougher for the square wave. The damage in the spall plane for the triangular wave is more localized meaning there is less bifurcation of cracks as they propagate across the spall plane of the sample. The thickness of the spalled region is indicated with a black and white bar, $h$ is take as the total length of the black ends of the bar while the white center portion only serves to guide the eye across the open spall plane.

is consistent with the statistical variation within a given sample reported by Vogler and Clayton [14], therefore being statistically insignificant for this brittle material. Moreover, this difference is an order of magnitude less than reported for the spall strength dependence on wave profile observed in ductile metals. For these two reasons any difference between the two samples is considered negligible.

Several differences can be seen in the optical micrographs of the recovered spalled samples (figure 4). The location of the spall plane within the thickness of the target is dramatically different, with it being centered for the square wave but near the rear of target for the triangular wave. This results from the difference in timing for interaction of the release waves off the back of the impactor and target because of the different impactor designs, as shown in figure 5. Of greater interest, the spall plane in both samples is relatively localized in the form of cracks consistent with brittle fracture. Relatively little plastic deformation is observed in the microstructure adjacent to the crack plane 

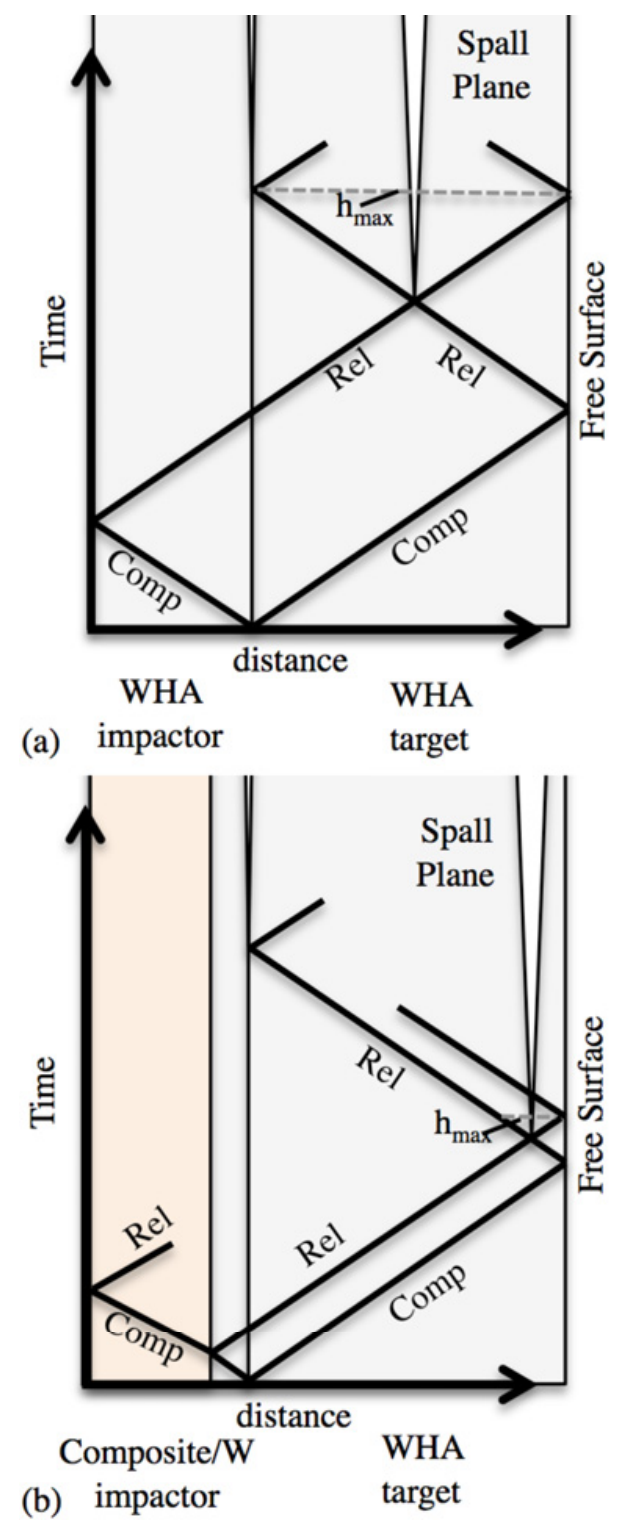

Fig. 5. $x$-t diagrams of compression (Comp) and release (Rel) waves for (a) the monolithic WHA impactor imparting a square wave and (b) the composite impactor imparting a triangular wave.

and no voids occur as is associated with incipient spall in ductile materials. In neither case are the cracks perfectly flat, but rather follow a path with roughness consistent with the length-scale of the tungsten particles. The squarewave resulted in a rougher or more circuitous spall plane and resulted in the formation of secondary cracks parallel to the primary spall plane. Conversely the damage in the spall plane for the triangular-wave is more localized with less bifurcation of cracks as they propagate across the spall plane of the sample. The bifurcating cracks are also consistent with brittle fracture. In this brittle material the time for crack nucleation is negligible compared to the duration of the experiment and the crack propagation rate is limited to the sound speed of the material (or in the case of the current experiments by the shock velocity). In dynamic fracture once a single crack is propagating at the sound speed it can no longer dissipate more
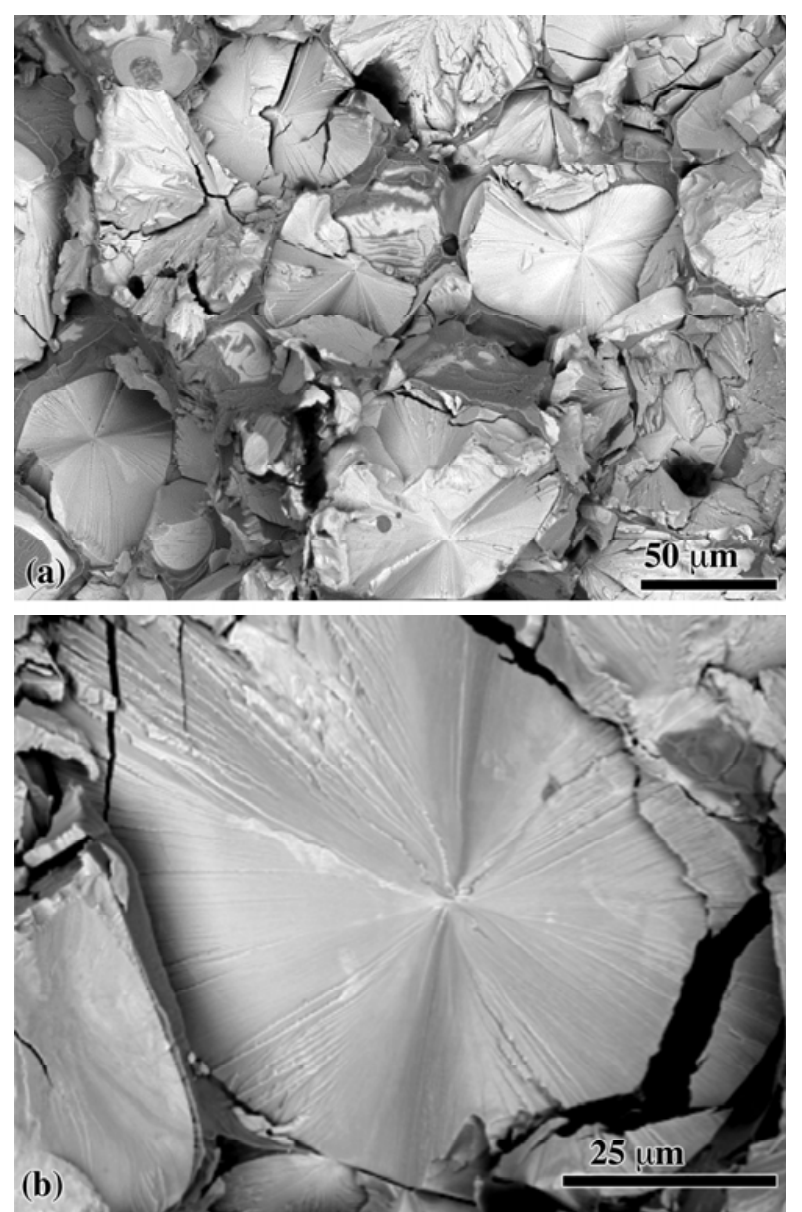

Fig. 6. SEM images of the fracture surface with cleavage as the preferred fracture mode for particle cracking.

energy by traveling faster, so instead it bifurcates to enable dissipation of more energy through the propagation of multiple cracks. Although the difference between square and triangular wave-forms does not significantly effect the spall strength of WHA, the difference in wave form does change the area under the curve effectively increasing the energy being put into the material. The WHA dissipates this additional energy through the generation of additional cracks.

Fracture surfaces of the spall planes are shown in figure 6 . The circuitous path observed in figure 4 is consistent with the length-scale of the tungsten particles. Both images show that cracks propagate by cleaving through the tungsten particles and linking up through the matrix. In addition to the primary crack plane through the particles, many of the particles exhibit extensive secondary cracking, a mechanism for further dissipating energy. Electron backscatter diffraction (EBSD) images, in figures 7 and 8 , further highlight the secondary cracks formed parallel to the primary crack. As observed in the optical micrographs there are more cracks that have linked up across multiple particles for the square-wave, both exhibit significant fracturing of stand alone particles where the crack does not appear to propagate into the matrix or surrounding particles. Subsurface damage of the brittle phase is often observed when mechanical toughening is achieved through 


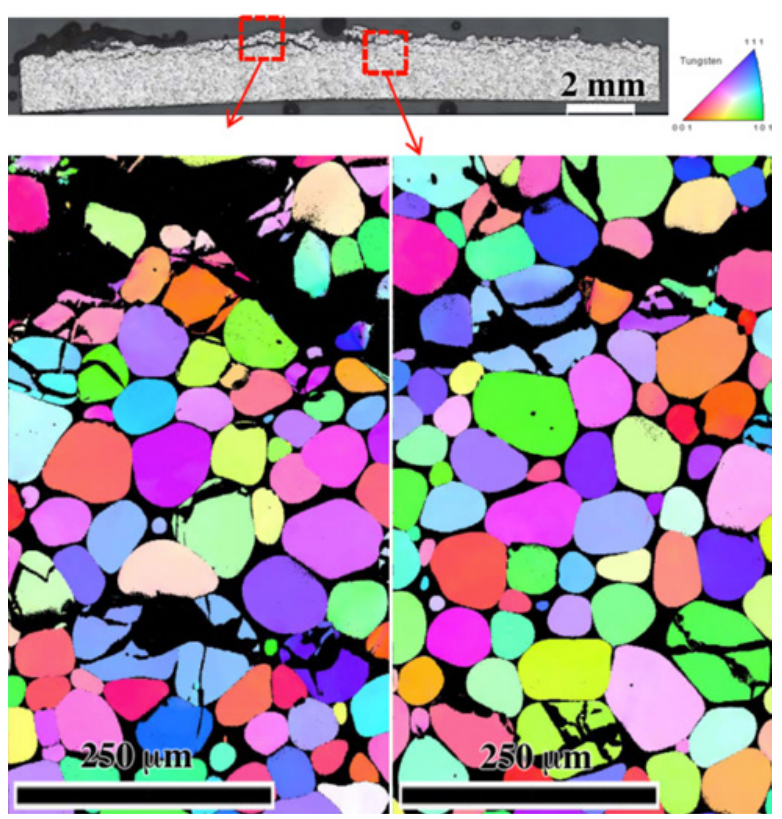

Fig. 7. EBSD scans near the spall plane of the square-wave loaded specimen. The shock direction is from bottom to top and the color indicates the particle's crystalline orientation with respect to the shock direction according to the color key.

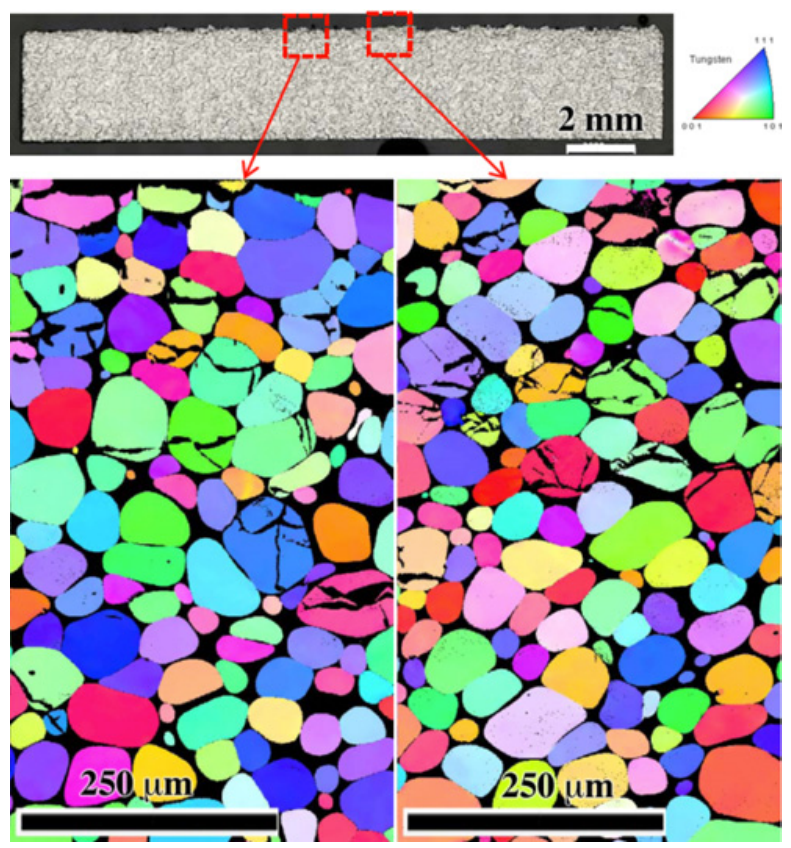

Fig. 8. EBSD scans near the spall plane of the triangle-wave loaded specimen. The shock direction is from bottom to top.

a multi-phase composite microstructure [22]. In figures 8 this diffuse isolated damage extends beyond the thickness of the spall region shown in figures 4(b). However, taking a larger value of $h$ further changes the calculated spall strength by less than a percent.

As discussed by Vogler and Clayton [14], the stress levels calculated are below the ideal tensile strength $(\sim 30 \mathrm{GPa})$ of a perfect tungsten lattice [23], but microscopic flaws within the $\mathrm{W}$ grains or along their edges may enable cleavage under the present loading conditions, particularly along $\left\{\begin{array}{lll}1 & 0 & 0\end{array}\right\}$ and $\left\{\begin{array}{lll}1 & 1 & 0\end{array}\right\}$ planes known to be preferred orientations for grain cleavage [24]. Such flaws may be created during processing or during the initial shock loading of the material. While simulations by Vogler and Clayton [14] suggested that initiation of spall fracture may be controlled by the interface between the particles and matrix, in agreement with experimental observations of [25], they hypothesized that crack propagation and macroscopic spall behavior would also require substantial grain cleavage. Once sufficiently large, a microcrack initiated along the boundary between a particle and matrix would propagate fully across the specimen, irrespective of the underlying microstructure. Consistent with this hypothesis the fracture surfaces shown in figure 6 indicate both inter-granular and cleavage mechanisms.

Further analysis of the EBSD images in figures 7 and 8 used projections of the normal of a given plane of a crack onto the respective pole figure of the particle measured that the particles preferentially cracked along the $\left\{\begin{array}{lll}0 & 0 & 1\end{array}\right\}$ and $\left\{\begin{array}{lll}1 & 0 & 1\end{array}\right\}$ planes. The current soft recovered spall data indicates that indeed grain cleavage is the dominant mechanism of dynamic damage in WHA although not along the expected plane from Gumbsch et al. [24].

In addition to the energy argument, another reason for the more constrained region damage in the case of the triangular-wave is simply the width of the shock. As stated earlier the square-wave remains within $95 \%$ of the peak stress for $0.52 \mu \mathrm{s}$, while the triangular wave profile remains within $95 \%$ of the peak stress for less than $0.08 \mu \mathrm{s}$. At a stress of $15.5 \mathrm{GPa}$ the shock velocity is $4.2 \mathrm{~mm} / \mu \mathrm{s}$ [11]. Therefore the shock width, and the width over which rarefaction waves can interact to form a spall plane, is $\sim 2.0$ and $0.3 \mathrm{~mm}$ for the square and triangular waves, respectively. For the square wave, the width of the shock is equivalent to the specimen thickness and will not constrain the thickness of the spall region. For the triangular wave, on the other hand, the shock width is equivalent to the thickness of the spall region, effectively indicating that maximum width of the overlapping rarefaction waves cannot extend any further. Interestingly, since EBSD exhibits the presence of isolated fractured particles beyond the thickness of the spall region as allowed by the shock width, they are unlikely to have fractured in tension due to the interaction of the release waves from the free surfaces of the target and impactor. Instead, they may have been fractured in compression during the initial shock loading either due directly to the applied compressive stress or due to the difference in mass impedance between particle and matrix generating localized states of tension.

\section{Conclusion}

Contrary to ductile metals where the kinetics for void initiation, growth, and linkage result in a strong dependence of spall strength on wave profile shape, the dynamic damage of tungsten heavy alloy (WHA) is seen to be relatively insensitive to changing from a square to triangular wave. The difference in spall strength for WHA at $\sim 15.5 \mathrm{GPa}$ 
was measured to be statistically insignificant for the two wave profiles considered in the current work. In both cases failure was by brittle cleavage through the tungsten particles and link up through the matrix resulting in a circuitous path on the length-scale of the particles. The difference in how energy is delivered to the specimen by the square wave led to an increase in the roughness or circuitousness of the crack plane and the initiation of parallel secondary cracks and crack bifurcation. While this enabled the brittle material to dissipate more energy, it did not change the strength of the material. Both samples exhibited isolated subsurface damage in the particles that had not linked up to form macroscale cracks. The particles were measured to have preferentially cracked along $\left\{\begin{array}{lll}0 & 0 & 1\end{array}\right\}$ and $\left\{\begin{array}{lll}1 & 0 & 1\end{array}\right\}$ planes. These results support the hypothesis that for brittle materials where all relevant damage kinetics occur on the time-scale of the shock velocity, the spall strength is shown to be dominated by the peak stress independent of loading profile.

\section{Acknowledgements}

Los Alamos National Laboratory is operated by LANS, LLC, for the NNSA of the US Department of Energy under contract DE-AC52-06NA25396. This research was supported under the auspices of the US Department of Energy and the Joint DoD/DOE Munitions Program.

\section{References}

1. G.T. Gray, N.K. Bourne, J.C.F. Millett, M.F. Lopez, Shock Compression of Condensed Matter - 2003, AIP Conf. Proc. 706, 461 (2004)

2. G.T. Gray, N.K. Bourne, B.L. Henrie, J. Appl. Phys. 101, 093507 (2007)

3. B.H. Sencer, S.A. Maloy, G.T.Gray III, Acta Materialia 53, 3293 (2005)

4. G.T. Gray, L.M. Hull, J.R. Faulkner, M.E. Briggs, E.K. Cerreta, F.L. Addessio, N.K. Bourne, Shock Compression of Condensed Matter - 2009, AIP Conf. Proc. 1195, 1097 (2009)

5. D.P. Dandekar, J. Appl. Phys 47, 4703 (1976).
6. Asay, J.R., Chhabildas, L.C., Dandekar, D.P., J. Appl. Phys 51, 4774 (1980)

7. A.K. Zurek, G.T. Gray III, J. De Physique IV Colloque C3, 631 (1991)

8. J.C.F. Millett, G. T. Gray III, N.K. Bourne, J. Appl. Phys. 101, 033520 (2007)

9. G.E. Hauver, The Hugoniot for $90 \mathrm{~W}-7 \mathrm{Ni}-3 \mathrm{Fe}$ tungsten alloy, ARBRL-MR-02987, U. S. Army Ballistic Research Laboratory Report Aberdeen Proving Ground, Maryland (1980)

10. P.J. Gaeta, D.P. Dandekar, Shock Waves in Condensed Matter-1987. Elsevier, 269 (1988)

11. D.P. Dandekar, W.J. Weisgerber, Int. J. Plasticity 15, 1291 (1999)

12. J.C.F. Millett, N.K. Bourne, Z. Rosenberg, J.E. Field, J. Appl. Phys. 86, 6707 (1999)

13. S. Bless, R. Chau, Shock Compression of Condensed Matter - 2005, AIP Conf. Proc. 603 (2006)

14. T.J. Vogler, J.D. Clayton, J. Mech. Phys. Solids 56, 297 (2008)

15. D.P. Dandekar, Int. J. Appl. Ceram. Technol. 1, 261 2004

16. S.N. Chang, J.H. Choi, Shock Compression of Condensed Matter - 1997, AIP Conf. Proc. 415 (1998)

17. Z. Baoping, Y. Chao, X. Yingming, J. Chunlan, Proceedings of IUTAM Symposium on Impact Dynamics 283 (1994)

18. G. T. Gray III, ASM Handbook, ASM International, Metals Park, OH, 8, 530 (2000)

19. S.A. Novikov, I.I. Divnov, A.G. Ivanov, Fizika Metallov i Metallovedenie 21, 608 (1966)

20. V.D. Gluzman and G.I. Kanel', Prikl. Mekh. Tekh. Fiz. 4, 146(1983)

21. G.I. Kanel', J. Appl. Mech. Tech. Phys. 42, 358 (2001) translated from Prikl. Mekh. Tekh. Fiz. 42, 194 (2001)

22. E.N. Brown, S.R. White, N.R. Sottos. J. Mater. Sci. 39, 1703 (2004)

23. D. Roundy, C.R. Krenn, M.L. Cohen, J.W. Morris, Philos. Mag. A-Phys. Condens. Matter. Struct. Defects Mech. Prop. 81, 1725 (2001)

24. P. Gumbsch, J. Riedle, A. Hartmaier, H.F. Fischmeister, Science 282, 1293 (1998)

25. T. Weerasooriya, Proceedings of the SEM Annual Conference on Experimental Mechanics 245 (2003) 